\title{
The Future of Climate Resilience in Wheat
}

\author{
Matthew Reynolds ${ }^{1,2}$, Janet Lewis ${ }^{1,2}$ \\ 1. International Maize and Wheat Improvement Center (CIMMYT) \\ 2. The Heat and Drought Wheat Improvement Consortium
}

December 13, 2021

This document is part of a series of short papers on "The Future of X", produced as part of foresight-related research supported by the CGIAR Research Program on Policies, Institutions, and Markets, and edited by Keith Wiebe (IFPRI) and Steven Prager (Alliance of Bioversity and (IAT). These short papers are intended to provide a focused, forward-looking perspective on key issues to support discussion on food, land, and water systems transformation. We thank two anonymous reviewers for their comments on an earlier draft.

This is an accepted version presented as a pre-print. It is currently undergoing final revision, editing, and production. A final version will be made available at http://foresight.cgiar.org.

\section{What is the issue?}

As the most widely cultivated crop globally - providing $20 \%$ of all human calories and protein there is an urgent need to increase wheat's resilience to harsher climates [1]. The risk of simultaneous crop failures due to heat and/or drought in global "breadbaskets" has risen and is projected to rise further [2-4]. Severe water scarcity events are predicted for up to $60 \%$ of the world's wheat-growing areas by the end of this century [5]. Furthermore, for each $1^{\circ} \mathrm{C}$ increase in average seasonal temperature, it is predicted that wheat yields will decrease by $6 \%$ on average globally, and much more in some already marginal environments where wheat is a traditional staple food $[6,7]$. At the current rate of yield gain, wheat production is predicted to fall well short of future demand due to population growth alone. Emerging environmental threats only make the challenge harder. On top of this, demand by consumers, farmers and the food industry is predicted to increase due to wheat's high grain-protein content relative to other cereals, wide growing range and adaptability to most environmental stresses. Since farmer adoption of improved cultivars is a critical part of adaptation [8], new and more targeted breeding efforts are needed to ensure that wheat's climate resilience is maximized [911].

\section{What research has been done?}

In collaboration with hundreds of public and private breeders that form part of the International Wheat Improvement Network (IWIN), several potential disease pandemics in wheat have been avoided through mining genetic resources to transfer disease resistance genes into modern cultivars [12,13]. More recently, physiological characterization of landraces and other genetic resources encompassing genes from wheat's wild relatives have provided outstanding sources of heat and drought tolerance traits [14-16]. Subsequent crossing and 
selection pilot programs at CIMMYT's research site in the Sonoran Desert showed that traits like deeper roots and high radiation use efficiency can be transferred from relatively exotic but stress-adapted genetic resources into agronomically modern wheat (Figure 1) [17-19]. The process can be accelerated using molecular tools to prioritize among available genetic resources, for example [20]. Additionally, remote sensing techniques permit high throughput phenotyping in realistic field environments at breeding scale [20-22].

\section{What has the research found?}

With support from national programs in South Asia and other climate vulnerable regions, novel germplasm encompassing climate resilience traits, derived from landraces and wild relatives, were tested against the best locally adapted lines. Lines encompassing novel sources of adaptive traits have shown outstanding performance under these multi-location field trials, even resulting in rapid release of cultivars in Pakistan and Afghanistan [19]. These outputs reveal that well-targeted crossing and selection strategies can overcome potential negative linkage drag, which is frequently observed when crossing elite lines with more exotic genomes such as the products of interspecific hybridization. Genetic bases for several of the traits have been identified in parallel studies [23-25]. For example, a common genetic basis was identified for adaptive root architecture under both heat and drought stress, respectively [26]. Other field studies have identified markers from exotic sources, such as re-synthesized hexaploids [18, 27].

\section{What gaps deserve further work?}

Although diversity of responses to heat and drought stress has been documented in wheat, and numerous genetic mapping studies have been conducted, individual studies are limited in scope and scale. Few resilience genes with consistent effect across genetic backgrounds and target environments have been identified $[28,29]$. While some physiological and metabolic factors for resilience have been reported [29], they represent just a handful of genotypes and condition. Due in large part to these knowledge gaps and concerns about linkage-drag and disrupting proven haplotypes/linkage-blocks, most breeders are still wary of crossing with more exotic sources. This is especially true when it comes to genetically complex traits that are harder to track, such as heat and drought tolerance.

However, as discussed, the targeted use of modern phenomic and genomic approaches to identify and breed with novel sources of climate resilience traits does pay off $[17,19,22,30]$. An example is success in the face of heat waves in Pakistan ( show considerable promise for a much larger and more targeted exploration of the World Wheat Collection, among others. The opportunity to explore and share the value of these largely untapped wheat genetic resource collections-approximately $0.8 \mathrm{~m}$ accessions are available worldwide- is one of the most powerful and efficient ways to adapt wheat to a changing climate [32-34]. This could also serve as a model than could be scaled out to other crops. 
However, such efforts require considerable knowledge and expertise to determine which combinations of traits and genes will be required to achieve climate resilience, as well as needed genetic gains. To prioritize the technologies most likely to help wheat adapt to ever harsher conditions, an expert consultation was organized by the CGIAR Research Program on Wheat (https://wheat.org/), involving more than 100 scientists from 77 research institutes in 32 developed and developing countries, whose suggestions were documented in 2015. Some of these ideas are already being validated and implemented by CIMMYT scientists and collaborators at the new translational research and breeding platform in the Sonoran Desert (the FFAR supported HeDWIC-Hub). Ties with the international network of disciplinary experts established under HeDWIC (http://www.hedwic.org/resources.html) and scientists in the Wheat Initiative's AHEAD Program( https://www.wheatinitiative.org/press-releases/aheadlaunched-in-berlin ) bring promising new ideas into focus in a timely way, along with other research networks like the International Wheat Yield Partnership https://iwyp.org/ that already shares technologies and infrastructure with the FFAR-HeDWIC Hub.

The proofs-of-concept presented here support the case for boosting the scope and scale of this approach, bringing breeding solutions to fruition more quickly in farmers' fields. New resources could also fast-track the application of promising advances from academia, by creating an opportunity for them to be validated in a realistic, field-based, context. This can be achieved, for example, by making competitive funds available to 'crowd-source' promising markers, screening technologies, genetic resources, etc. These contributions, combined with CIMMYT's experience of field research and breeding, would lead to numerous tangible outputs (in roughly chronological order):

- Identification of new genetic resources encompassing previously undiscovered sources of heat and drought tolerance traits/alleles.

- New or refined field-based screening and bioinformatics protocols that can interpret data collected on lines showing highly diverse morphology (as typical of genetic resources).

- New breeding schemes that overcome the challenges of breeding with exotic sources through strategic trait-based crossing and selection. Accelerated proofs-of-concept for the most promising crossing strategies could also be achieved through rapid cycle prebreeding.

- Ensuring that novel climate-resilience traits will not diminish consumer preferencerelated traits, considering the diverse uses of wheat for making bread, pasta, chapatis, baking, etc.

- Delivery of the most successful germplasm and technologies, and their scale-out, via the IWIN -a testing network that includes hundreds of public and private breeders in 90 countries worldwide - whose impacts and continued value to farmers and urban consumers is unparalleled in crop improvement [31].

- Comprehensive physiological and genetic dissection of exceptional genotypes to strategize their use in resilience breeding. 
- More systematic understanding of wheat's genetic potential to adapt to heat and drought scenarios, and new, validated methodologies to exploit this in climate resilience breeding.

Foresight and related approaches can help us front run some of these challenges. Based on the proofs of concept already delivered, the approach has significant promise for scale-out, and could serve as a model for cereals and other crops facing unpredictable and harsher climates. Furthermore, translational research and pre-breeding could also achieve a significant boost by linking to upstream discovery research (e.g. via public-private partnerships) that aims to fill significant knowledge gaps that currently limit a more comprehensive understanding of yield and climate resilience in most crops ${ }^{35}$.

Even with the approach outlined here, the process takes time. Crop Design is an ongoing process (Figure 1). Screening Genetic Resources can take a year or two (using Phenotyping and Genetic Analysis); Crossing and Selection typically takes between 3-5 years; Evaluation of genetic gains through international testing is usually conducted in one season through the International Wheat Improvement Network (IWIN). Dissemination and adoption take time as well. The cumulative lag until farmer adoption at scale, combined with rapidly changing resource and market conditions, means it is critical that the process be designed with future conditions in mind.

\section{PHySIOLOGIGAL PRE-BREEDING PIPELINE}

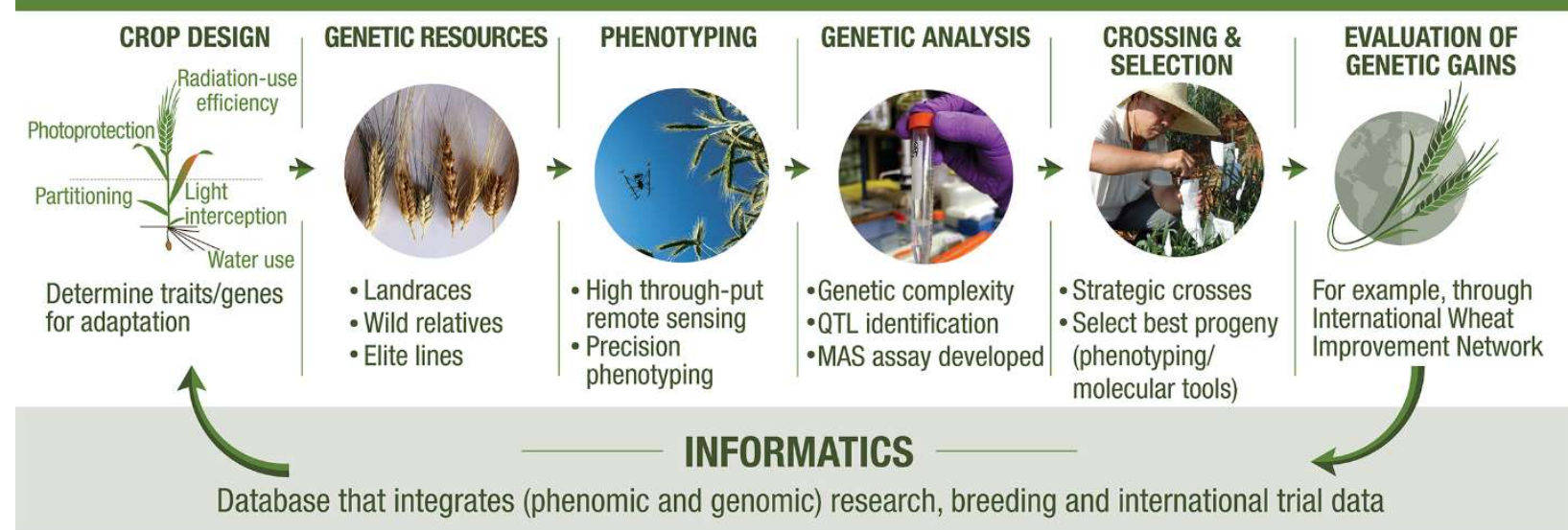

Fig. 1. Main research steps involved in translating promising technologies into genetic gains (Graphical abstract ${ }^{30}$ ). Reprinted under creative commons license https://creativecommons.org/licenses/by-nc-nd/4.0/. 


\section{Links to more information}

- HeDWIC website: http://www.hedwic.org/

- HeDWIC video: https://www.youtube.com/watch?time continue $=1 \& v=G c N n x X T$ Tugpo\&feature $=e m b$ tit $\underline{\text { le }}$

\section{References}

1. Shiferaw, B. et al. Crops that feed the world 10. Past successes and future challenges to the role played by wheat in global food security. Food Secur. 5, 291-317 (2013).

2. Sarhadi, A., Ausín, M. C., Wiper, M. P., Touma, D. \& Diffenbaugh, N. S. Multidimensional risk in a nonstationary climate: Joint probability of increasingly severe warm and dry conditions. Sci. Adv. 4, eaau3487 (2018).

3. Kornhuber, K. et al. Amplified Rossby waves enhance risk of concurrent heatwaves in major breadbasket regions. Nat. Clim. Chang. 10, 48-53 (2020).

4. Gaupp, F., Hall, J., Hochrainer-Stigler, S. \& Dadson, S. Changing risks of simultaneous global breadbasket failure. Nat. Clim. Chang. 10, 54-57 (2020).

5. Trnka, M. et al. Mitigation efforts will not fully alleviate the increase in water scarcity occurrence probability in wheat-producing areas. Sci. Adv. 5, 1-12 (2019).

6. Liu, B. et al. Similar estimates of temperature impacts on global wheat yield by three independent methods. Nat. Clim. Chang. 6, 1130-1137 (2016).

7. Zhao, C. et al. Temperature increase reduces global yields of major crops in four independent estimates. Proc. Natl. Acad. Sci. 114, 9326-9331 (2017).

8. Challinor, A. J. et al. A meta-analysis of crop yield under climate change and adaptation. Nat. Clim. Chang. 4, 287-297 (2014).

9. Ray, D. K., Mueller, N. D., West, P. C. \& Foley, J. A. Yield Trends Are Insufficient to Double Global Crop Production by 2050. PLoS One 8, e66428 (2013).

10. Gourdji, S. M., Mathews, K. L., Reynolds, M., Crossa, J. \& Lobell, D. B. An assessment of wheat yield sensitivity and breeding gains in hot environments. Proc. R. Soc. B 280, 20122190 (2013).

11. Ray, D. K., Ramankutty, N., Mueller, N. D., West, P. C. \& Foley, J. A. Recent patterns of crop yield growth and stagnation. Nat. Commun. 3, 1-7 (2012).

12. Trethowan, R. M. \& Mujeeb-Kazi, A. Novel germplasm resources for improving environmental stress tolerance of hexaploid wheat. Crop Sci. 48, 1255-1265 (2008).

13. Ortiz, R. et al. Wheat genetic resources enhancement by the International Maize and Wheat Improvement Center (CIMMYT). Genet. Resour. Crop Evol. 55, 1095-1140 (2008). 
14. Reynolds, M., Dreccer, F. \& Trethowan, R. Drought-adaptive traits derived from wheat wild relatives and landraces. J. Exp. Bot. 58, 177-186 (2007).

15. Lopes, M. S. \& Reynolds, M. P. Drought adaptive traits and wide adaptation in elite lines derived from re-synthesized hexaploid wheat. Crop Sci. 51, 1617-1626 (2011).

16. Cossani, C. M. \& Reynolds, M. P. Heat stress adaptation in elite lines derived from synthetic hexaploid wheat. Crop Sci. 55, 2719-2735 (2015).

17. Pask, A. et al. A wheat phenotyping network to incorporate physiological traits for climate change in South Asia. F. Crop. Res. 168, 156-167 (2014).

18. Molero, G. et al. Elucidating the genetic basis of biomass accumulation and radiation use efficiency in spring wheat and its role in yield potential. Plant Biotechnol. J. 17, 12761288 (2019).

19. Reynolds, M. P. et al. Strategic crossing of biomass and harvest index-source and sinkachieves genetic gains in wheat. Euphytica 213, 257 (2017).

20. Sansaloni, C. et al. Diversity analysis of 80,000 wheat accessions reveals consequences and opportunities of selection footprints. Nat. Commun. 11, 4572 (2020).

21. Tattaris, M., Reynolds, M. P. \& Chapman, S. C. A Direct Comparison of Remote Sensing Approaches for High-Throughput Phenotyping in Plant Breeding. Front. Plant Sci. 7, 1-9 (2016).

22. Reynolds, M. et al. Breeder Friendly Phenotyping. Plant Sci. 295, 110396 (2020).

23. Liu, C. et al. Genetic dissection of heat and drought stress QTLs in phenology-controlled synthetic-derived recombinant inbred lines in spring wheat. Mol. Breed. 39, 1-18 (2019).

24. Li, L. et al. Genetic dissection of drought and heat-responsive agronomic traits in wheat. Plant. Cell Environ. 42, 2540-2553 (2019).

25. Pinto, R. S., Lopes, M. S., Collins, N. C. \& Reynolds, M. P. Modelling and genetic dissection of staygreen under heat stress. Theor. Appl. Genet. 129, 2055-2074 (2016).

26. Pinto, R. S. \& Reynolds, M. P. Common genetic basis for canopy temperature depression under heat and drought stress associated with optimized root distribution in bread wheat. Theor. Appl. Genet. 128, 575-585 (2015).

27. Rosyara, U. et al. Genetic Contribution of Synthetic Hexaploid Wheat to CIMMYT's Spring Bread Wheat Breeding Germplasm. Sci. Rep. 9, 1-11 (2019).

28. Acuña-Galindo, M. A., Mason, R. E., Subramanian, N. K. \& Hays, D. B. Meta-analysis of wheat QTL regions associated with adaptation to drought and heat stress. Crop Sci. 55, 477-492 (2015).

29. Gupta, P., Balyan, H. \& Gahlaut, V. QTL Analysis for Drought Tolerance in Wheat: Present Status and Future Possibilities. Agronomy 7, 5 (2017). 
30. Reynolds, M. \& Langridge, P. Physiological breeding. Curr. Opin. Plant Biol. 31, 162-171 (2016).

31. Reynolds, M. P. et al. Improving global integration of crop research. Science (80-. ). 357, 359-360 (2017).

32. Varshney, R. K., Singh, V. K., Kumar, A., Powell, W. \& Sorrells, M. E. Can genomics deliver climate-change ready crops? Curr. Opin. Plant Biol. 45, 205-211 (2018).

33. Rai, N. et al. Marker-assisted backcross breeding for improvement of drought tolerance in bread wheat (Triticum aestivum L. em Thell). Plant Breed. 137, 514-526 (2018).

34. Crain, J., Mondal, S., Rutkoski, J., Singh, R. P. \& Poland, J. Combining High-Throughput Phenotyping and Genomic Information to Increase Prediction and Selection Accuracy in Wheat Breeding. Plant Genome 11, 170043 (2018).

35. Reynolds, M. et al. Addressing Research Bottlenecks to Crop Productivity. Trends Plant Sci. 26, 607-630 (2021). 